\title{
SUPPLEMENT TO THE BIBLIOGRAPHY OF THE DE- SCRIBED LIFE-HISTORIES OF THE RHOPALOCERA OF AMERICA NORTH OF MEXICO
}

\author{
By V. G. Dethier \\ Ohio State University
}

In the Bibliography of the Described Life-Histories of the Rhopalocera of America North of Mexico 1889-1937 (Davenport and Dethier, 1938) an attempt was made to bring up to date the section on Rhopalocera of the Bibliographical Catalogue of Henry Edwards (1889). During the eight years that have elapsed since the appearance of the former not a few new life histories have been described. Lacunae in the descriptions of previously known life histories have also been filled. It is felt that a supplement at this time may be of assistance to those engaged in this field of study. Accordingly the following list patterned after the 1938 bibliography is offered. McDunnough's (1938) check list, which was published after the appearance of the bibliography, is now followed.

For additional data on feeding habits the reader is referred to Comstock (1927), Wolcott (1936), Field (1938), and Hayward (1941).

Papilio polydamas L. Dethier, V. G. Psyche, 47(1): 22-23, 1940 (larva).

Food plant: Aristolochia argyreoneuron, A. ringens, $A$. forckeli, $A$. redicula, $A$. saccata, $A$. tagala, $A$. fimbriata, $A$. brasiliensis, A. cymbifera, $A$. elegans, A. gigantea, $A$. grandiflora, A. glandulosa, A. hians, A. indica, A. Kaempferi, A. Roxburghiana, A. Ruiziana, A trilobata.

P. ajax americus Koll. Brown, F. M. Bul. S. Calif. Acad. Sci., 38(3): 200-201, 1939 (larva, chrysalis).

Food plant: "white carrot."

P. celadon Luc. Dethier, V. G. Psyche, 47(1): 22, 1940 (egg).

Phoebis sennae marcellina Cram. Tilden, J. W. Pan.-Pac. Ent., 20: 117, 1944 (life history).

Food plant: Cassia tomentosa. 
P. agarithe Bdv. Wolcott, G. N. Jour. Agri. Univ. Puerto Rico, 25(2): 123, 1941 (food plant).

Food plant: Pithecolobium dulcis.

Eurema lisa Bdv. \& Lec. Dethier, V. G. Psyche, 47(1): 21-22, 1940 (larva).

Food plant: Mimosa pudica.

Nathalis iole Bdv. Dethier, V. G. Psyche, 47(1): 19-21, 1940 (life history).

Food plant: Bidens leucantha.

Ascia monuste L. Wolcott, G. N. Jour. Agri. Univ. Puerto Rico, 25(2): 123, 1941 (food plant).

Food plant: Cleome gynandra, Allium.

Danaus menippe Hbn. Wolcott, G. N. Jour. Agri. Univ. Puerto Rico, 25(2): 122, 1941 (food plant).

Food plant: Asclepias curassavica.

D. berenice strigosa Bates Dammers, C. M. Bul. S. Calif. Acad. Sci., 36(1): 23-24, pl. 10, 1937 (food plant).

Food plant: Philibertia heterophylla, Stapelia.

Argynnis nokomis Edw. Comstock, J. A. Bul. S. Calif. Acad.

Sci., 39(1): 76, 77, pl. 8, 1940 (egg).

Food plant: Viola.

A. leto Behr Comstock, J. A. Bul. S. Calif. Acad. Sci., 39(1): 75-77, pl. 8, 1940 (egg, larva).

Food plant: Viola.

A. hydaspe viridicornis Comst. Comstock, J. A. Bul. S. Calif. Acad. Sci., 39(1): 76, 77, pl. 8, 1940 (egg).

Food plant: Viola.

A. callippe comstocki Gund. Comstock, J. A. Bul. S. Calif. Acad. Sci., 39(1): 76, 77, pl. 8, 1940 (egg).

Food plant: Viola.

Euphydryas chalcedona Dbldy. \& Hew. Dammers, C. M. Bul. S. Calif. Acad. Sci., 39(2) : 123-125, 1940 (food plant).

Food plant: Scrophularia antirrhinoides Benth., Buddleia.

E. cooperi Behr Doudoroff, M. Canad. Ent., 69(5): 117-118, 1937 (larva).

E. gillettii Barnes Comstock, J. A. Bul. S. Calif. Acad. Sci., 39(2): 111-113, pl. 20, 1940 (egg, larva).

Food plant: Lonicera involucrata Banks.

Junonia genoveva Cram. Wolcott, G. N. Jour. Agri. Univ. Puerto Rico, 20(1): 399, 1936 (larva, chrysalis).

Food plant: Valerianoides jamaicensis. 
Anartia jatrophae Joh. Wolcott, G. N. Jour. Agri. Univ. Puerto Rico, 20(1): 399, 1936 (larva, chrysalis).

Food plant: Bacopa monniera.

Anartia jatrophae jamaicensis Moesch. Dethier, V. G. Psyche, 48(2/3): 71-73, pls. 5-6, 1941 (egg, larva).

Food plant: Lippia.

Victorina steneles L. Wolcott, G. N. Jour. Dept. Agric. Puerto Rico, 7(1): 1924 (larva, chrysalis).

Food plant: Blechum brownei.

Historis orion Fabr. Smyth, E. G. Fourth Rept. Bd. Comm. Agri. Puerto Rico, 1914-15: 45-50, 1916 (larva, chrysalis). Wolcott, G. N. Jour. Agri. Univ. Puerto Rico, 20(1): 401, 1936 (larva, chrysalis).

Food plant: Cecropia peltata.

Eumaeus atala Poey Dethier, V. G. Psyche, 48(2/3): 75, pl. 6, 1941 (larva, chrysalis).

Mitoura spinetorum Hew. Comstock, J. A. and Dammers, C. M.

Bul. S. Calif. Acad. Sci., 37(1) : 30-32, pls. 3-4, 1938 (life history).

Food plant: Arceuthobium campylopodum Engelm.

Leptotes theonus Luc. Wolcott, G. N. Jour. Agri. Univ. Puerto Rico, 25(2): 123, 1941 (food plant).

Food plant: Crotalaria incana.

Hemiargus hanno Stoll. Dethier, V. G. Psyche, 47(1): 24, 1940( egg, larva). Wolcott, G. N. Jour. Agri. Univ. Puerto Rico, 25(2): 123, 1941 (food plant).

Food plant: Mimosa pudica, Macroptilium lathyroides.

Polygonus amyntas Fabr. Wolcott, G. N. Jour. Agri. Univ. Puerto Rico, 20(1) : 407, 1936 (larva). Wolcott, G. N. Jour. Agri. Univ. Puerto Rico, 25(2): 124, 1941 (food plant). Dethier, V. G. Psyche, 49 (1/2) : 5-6, 1942 (larva).

Food plant: Ichthyomethia piscipula, Lonchocarpus, Derris eliptica.

Proteides idas Cram. Wolcott, G. N. Jour. Agri. Univ. Puerto Rico, 25(2): 124, 1941 (food plant).

Food plant: Derris eliptica.

Urbanus dorantes Stoll. Dethier, V. G. Psyche, 49(1/2): 4-5, 1942 (egg, larva).

Food plant: Clitorea and related genera.

Cabares potrilla Luc. Dethier, V. G. Psyche, 47(1): 24-25, pl. 3, 1940 (egg). 
Pyrgus syrichtus Fabr. Dethier, V. G. Psyche, 47(1): 24, pl. 3, 1940; 49(1/2): 6-7, 1942 (life history).

Food plant: Sida.

P. communis occidentalis Skin. Dethier, V. G. Bul. S. Calif. Acad. Sci., 43(1) : 30-32, pl. 11, 1944 (life history).

Food plant: Sidalcea.

Achlyodes thraso Hbn. Wolcott, G. N. Jour. Dept. Puerto Rico, $7(1): 1924$ (larva, chrysalis).

Food plant: Citrus, Zanthoxylum monophyllum.

Ancyloxypha numitor Fabr. Dethier, V. G. Canad. Ent., 71: 118, 1939 (larva). Dethier, V. G. Bul. S. Calif. Acad. Sci., 37(2): 74-77, pls. 18-20, 1938 (life history).

Food plant: Grasses.

Hesperia juba Scud. Lindsey, A. W. Denison Univ. Bul., Jour.

Sci. Lab., 20: 121-125, pl. 16, 1923 (egg, larva).

Food plant: Grasses.

H. leonardus Harr. Dethier, V. G. Canad. Ent., 71: 118, 1939 (egg, larva).

Food plant: Grasses.

Polites verna Edw. Dethier, V. G. Canad. Ent., 71: 117-118, 1939 (egg, larva).

Food plant: Grasses.

P. manataaqua Harr. Dethier, V. G. Canad. Ent., 70: 255, 1938 (egg, larva). Dethier, V. G. Bul. S. Calif. Acad. Sci., 40(2) : 109-111, pls. 8-9, 1941 (larva).

Food plant: Grasses.

P. themistocles Latr. Dethier, V. G. Canad. Ent., 70: 255-256, 1938 (egg, larva). Dethier, V. G. Bul. S. Calif. Acad. Sci., 41(1): 41-43, pl. 9, 1942 (larva, chrysalis).

Food plant: Grasses.

P. baracoa Luc. Dethier, V. G. Psyche, 46(4) : 148-149, pl. 9, 1939 (egg, larva).

Food plant: Grasses.

P. peckius Kby. Dethier, V. G. Bul. S. Calif. Acad. Sci., 38(3): 188-190, pl. 50, 1939 (life history).

Food plant: Grasses.

P. sabuleti Bdv. Dethier, V. G. Bul. S. Calif. Acad. Sci., 42(3): 128-131, pl. 13, 1943 (life history).

Food plant: Grasses. 
P. mystic Scud. Dethier, V. G. Canad. Ent., 70: 256-257, 1938 (egg, larva). Dethier, V. G. Bul. S. Calif. Acad. Sci., 39(2): 108-110, pl. 19, 1940 (larva, chrysalis).

Food plant: Grasses.

Catia ortho egeremet Scud. Dethier, V. G. Canad. Ent., 70: 257-258, 1938 (egg, larva).

Food plant: Grasses.

Poanes hobomok Harr. Dethier, V. G. Canad. Ent., 70: 258259, 1938 (egg, larva).

Food plant: Grasses.

P. radians Luc. Dethier, V. G. Psyche, 46(4): 150-151, pl. 9, 1939 (egg, larva).

Food plant: Grasses.

$P$. haitensis Skin. Wolcott, G. N. Jour. Dept. Agri. Puerto Rico, 5(2): 1-47, 1921 (life history).

Food plant: Sugar cane.

Lerodea tripunctus H.-S. Dethier, V. G. Psyche, 46(4): 151152, pl. 9, 1939 (larva, chrysalis). Dethier, V. G. Mem. Soc. Cubana Hist. Nat., 16(2): 167-176, pl. 26, 1942 (life history).

Food plant: Grasses, sugar cane.

Panoquina sylvicola H.-S. Jones, T. H. and Wolcott, G. N. Jour. Dept. Agri. Puerto Rico, 6(1): 38-50, fig. 11, 1922 (life history). Wolcott, G. N. Jour. Dept. Agri. Puerto Rico, 6(1) : 32-37, 1922 (larva, chrysalis). Wolcott, G. N. Jour. Agri. Univ. Puerto Rico, 20(1): 411, 1 fig., 1936 (larva). Dethier, V. G. Psyche, 46(4): 152-153, pl. 9, 1939 (egg, larva). Dethier, V. G. Mem. Soc. Cubana Hist. Nat., 16(2): 167-176, pl. 26, 1942 (life history).

Food plant: Grasses, sugar cane, bamboo, rice.

P. nyctelius Latr. Jones, T. H. and Wolcott, G. N. Jour. Dept. Agri. Puerto Rico, 6(1): 38-50, figs. 9, 15, 1922 (life history). Wolcott, G. N. Jour. Dept. Agri. Puerto Rico, 6(1): 32-37, 1922 (larva). Wolcott, G. N. Jour. Agri. Univ. Puerto Rico, 20(1) : 410, 1 fig., 1936 (larva). Dethier, V. G. Psyche, 46(4): 153-154, pl. 9, 1939 (larva, chrysalis). Dethier, V. G. Mem. Soc. Cubana Hist. Nat., 16(2): 167176, pl. 26, 1942 (life history).

Food plant: Grasses, sugar cane. 


\section{REFERENCES}

Comstock, J. A. 1927. Butterflies of California.

Davenport, D. and Dethier, V. G. 1938. Bibliography of the described life-histories of the Rhopalocera of America north of Mexico 1889-1937. Ent. Amer. $17(4): 155-194$.

Edwards, H. 1889. Bibliographical catalogue of the described transformations of North American Lepidoptera. Bul. U. S. Nat. Mus. No. 35: 1-37.

Field, W. D. 1938. A manual of the butterflies and skippers of Kansas. Bull. Univ. Kansas 39(10): 1-328.

Hayward, K. J. 1941. Plantas alimenticias de Hesperidos Argentinos. Rev. Soc. Ent. Argentina 11(1): 31-36.

McDunnough, J. 1938. Check list of the Lepidoptera of Canada and the United States of America. Part I Macrolepidoptera. Mem. S. Calif. Acad. Sci. 1: $1-36$.

Wolcott, G. N. 1936. "Insectæ Borinquenses." Jour. Agri. Univ. Puerto Rico 20(1): 396-412, 602-627.

\section{DICHELONYX CANADENSIS HORN}

By C. A. Frost

Framingham, Mass.

In his monograph of this genus in the Trans. Am. Ent. Soc. Vol. XXVII, 1901, Mr. H. C. Fall writes, "This species is quite unknown to me nor do I know if there are any in collections besides the type." The only locality given in Leng's list is "Can." which is evidently the locality given by Horn in his description in 1876.

Specimens in my collection taken by me at Paris, Maine are dated: - June 17, 1912, July 15, 1913, June 10, 1925, June 30, 1932 , July 6,1933 . and July 7, 1937. Some of them are labelled as swept from Corylus rostrata, or taken on the leaves of this plant where they were observed feeding. On June 22, 1945, while eating lunch in the shade of a large white pine on the edge of an open pasture, I noticed a couple of specimens on the grass stems in front of me. Later some unexplained desire to enter the dense shadows of the pines and hemlocks behind me was acted on. There I found this beetle flying over the forest floor in great numbers; they flew from one to three feet high frequently coming to rest on the ground or on fallen twigs and branches, for a few moments only in many cases; no copulation was observed. 106 specimens were taken and their numbers seemed to be but little diminished at the end of an hour, which 

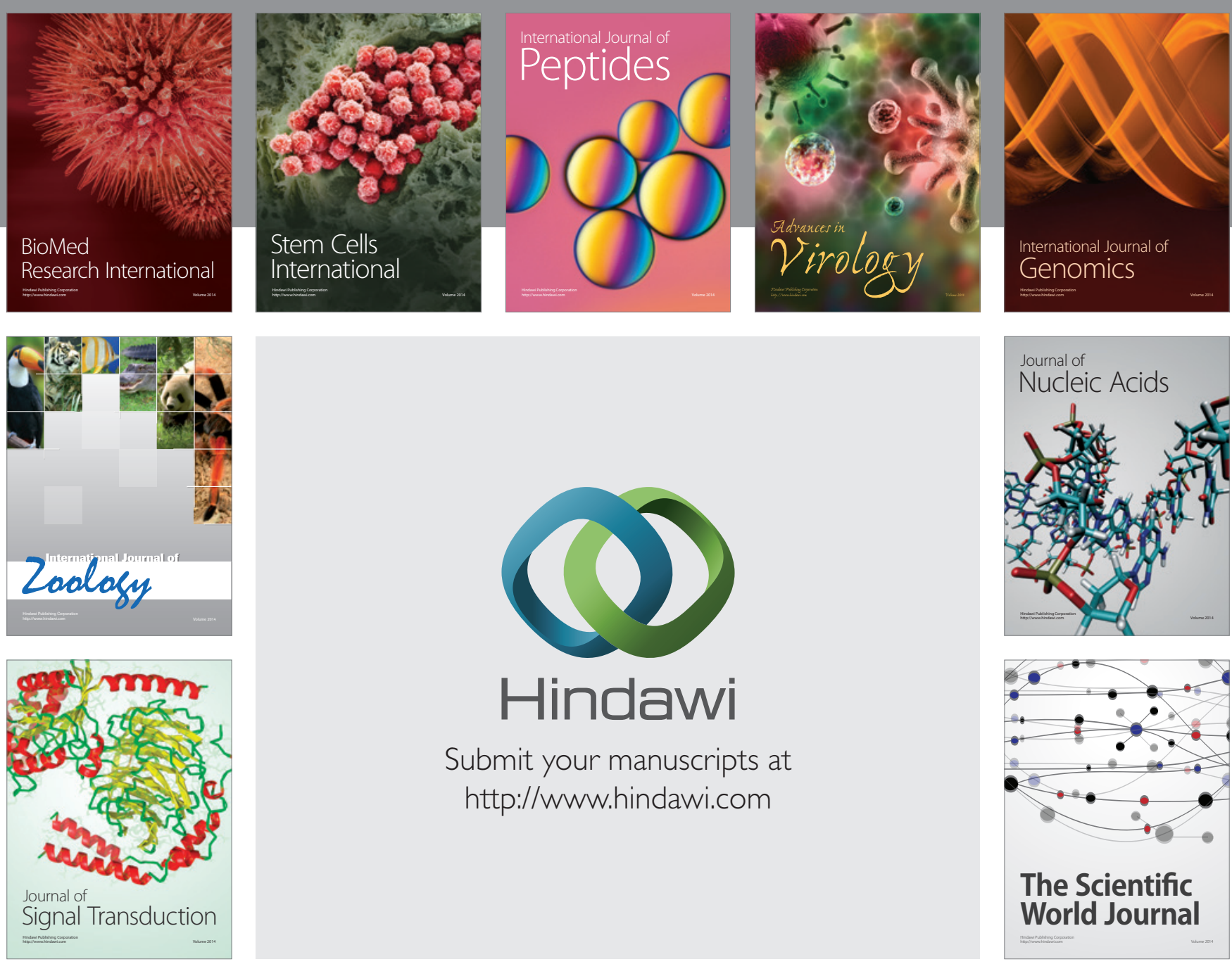

Submit your manuscripts at

http://www.hindawi.com
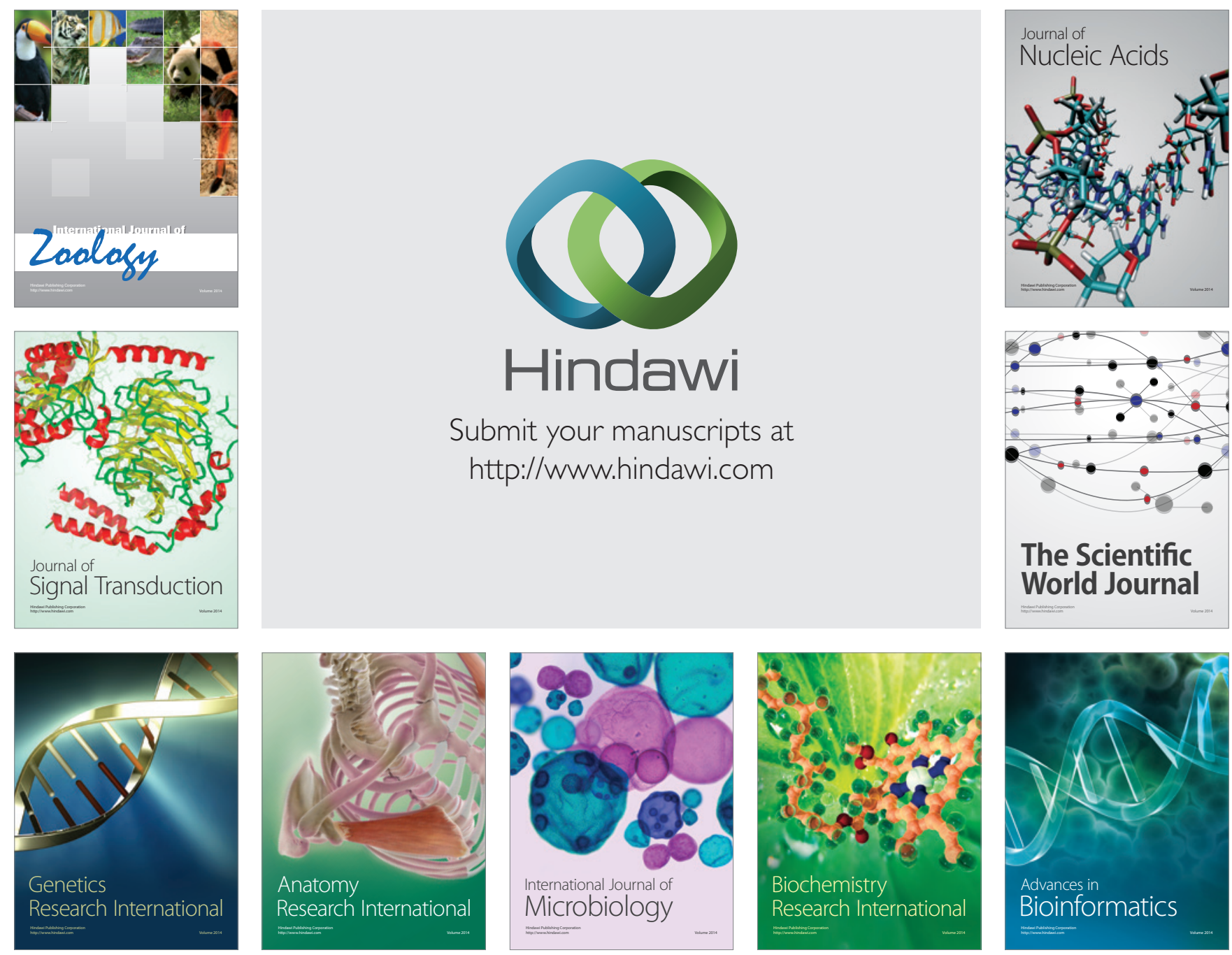

The Scientific World Journal
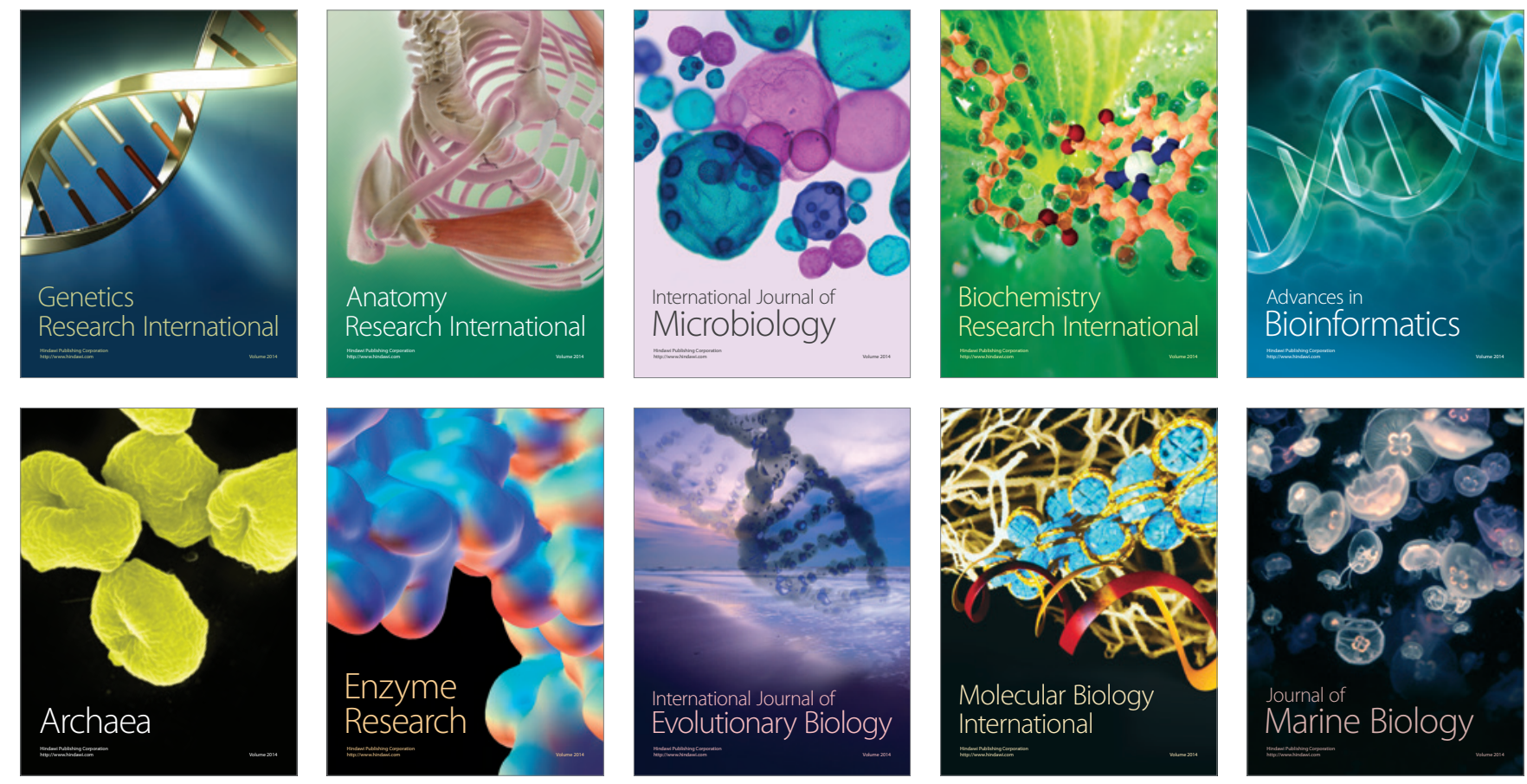